\title{
Primary Care Providers' Views on a Future Lung Cancer Screening Program
}

\author{
Mary Ann O'Brien ${ }^{a}$ \\ Diego Llovet ${ }^{\mathrm{b}, \mathrm{c}}$ \\ Frank Sullivan ${ }^{\text {a,d-f }}$ \\ Lawrence Paszat d,g,h
}

Affiliations:

a Department of Family and Community Medicine, University of Toronto, Toronto, Canada

b Cancer Care Ontario, Toronto, Canada

c Institute of Health Policy, Management and Evaluation, University of Toronto, Toronto, Canada

d Dalla Lana School of Public Health, University of Toronto, Toronto, Canada

e UTOPIAN Practice-based Research Network, University of Toronto, Toronto, Canada

f University of St. Andrews, United Kingdom

g Institute for Clinical Evaluative Sciences, Toronto, Canada

h Sunnybrook Research Institute, Toronto, Canada

Corresponding Author:

Mary Ann O’Brien, PhD

Department of Family \& Community Medicine

500 University Avenue, Fifth Floor 
Toronto, ON M5G 1 V7

Phone: 416 978-7994

Email: maryann.obrien@utoronto.ca

Running head: Primary Care Providers' Views on a Future Lung Cancer Screening Program

Key words: early detection of cancer; family practice; qualitative research 
Key Messages

- Variable primary care provider preferences for role in lung cancer screening

- Primary care providers want information about lung cancer screening

- Allied health professionals may have role in assessing eligibility for screening

- Smoking cessation is a key strategy in any lung cancer screening program 


\section{Abstract}

Background: The National Lung Screening Trial demonstrated that screening with low dose computed tomography significantly reduces mortality from lung cancer in high risk individuals.

Objective: To describe the role preferences and information needs of primary care providers in a future organized lung cancer screening program.

Methods: We purposively sampled primary care providers from diverse health regions of Ontario and from different practice models including family health teams and community health centres. We also recruited family physicians with a leadership role in cancer screening. We used focus groups and a nominal group process to identify informational priorities. Two analysts systematically applied a coding scheme to interview transcripts.

Results: Four groups were held with 33 providers and administrative staff (27 (82\%) female; 21 (64\%) physicians; 6 (18\%) other health professionals; 6 (18\%) administrative staff). PCPs and staff were generally positive about a potential lung cancer screening program but had variable views on their involvement. Informational needs included evidence of potential benefits and harms of screening. Most providers preferred that a new program be modelled on positive features of an existing breast cancer screening program. Lung cancer screening was viewed as a new opportunity to counsel patients about smoking cessation.

Conclusions: The development of a future lung cancer screening program should consider the wide variability in the roles that primary care providers preferred. An explicit link to existing smoking cessation programs was seen as essential. As providers had significant information needs, learning materials and opportunities should be developed with them.

Word count 247; limit: 250 


\section{[need to reduce manuscript by 200 words)}

\section{Introduction}

Lung cancerhas a poor prognosis with approximately $10 \%$ five-year relative survival rate for Stage IVa that is primarily due to late detection. Prognosis improves if cancer is found at an early stage. The relative survival for Stage la and Ib non-small cell lung cancer is $92 \%$ and $68 \%$ respectively (1).

The National Lung Screening Trial (NLST) in the United States (2) demonstrated and the Lung Cancer Screening Trial Pilot in the United Kingdom (3) suggested that screening with low dose CT scans (LDCT) significantly reduces mortality from lung cancer among high risk individuals aged 55 to 74 years as compared to chest x-rays. In the NLST, there were potential harms associated with LDCT screening (4). Averaged across three annual lung scans, $11.2 \%$ of screens were followed by an extra scan (with additional radiation), $0.9 \%$ of scans led to diagnosis of lung cancer (10-15\% of which would not have appeared during the patient's remaining life), $0.5 \%$ of scans led to biopsy or bronchoscopy that did not find cancer, and $0.3 \%$ of scans led to surgery that did not reveal lung cancer.

Guidelines in Canada and the United States now recommend LDCT screening for high risk individuals (58). In Ontario, primary care physicians are instrumental in screening for other types of cancer, specifically cervical, colorectal, breast cancer. For cervical cancer screening, physicians perform pap smears; for colorectal cancer screening, they distribute fecal occult blood test kits and / or refer for colonoscopy; and in some settings, they refer for screening mammography.

(https://www.cancercareontario.ca/en/get-checked-cancer). For primary care providers (PCPs), there may be challenges in identifying patients who are eligible for LDCT screening since criteria include duration and amount of smoking as well as age (5-8). Although several US studies have reported PCP interest in LDCT screening $(9,10)$, we did not identify Canadian data on PCP preferences for involvement in an organized LDCT screening program. 
The purpose of the present study was to describe the views of PCPs and staff members on their potential roles and informational needs in an organized LDCT screening program. We were also interested in whether PCPs would be willing and able to identify screen-eligible persons and counsel interested persons regarding potential benefits and harms of LDCT screening. At the time of this study, an organized LDCT screening program for lung cancer was not available in Ontario although pilot work for a potential program is underway (https://www.cancercare.on.ca/pcs/screening/other_cancers/).

\section{Methods}

This descriptive qualitative study (11) was one of five components of a health technology assessment on LDCT screening in Ontario, Canada (Principal Investigator: L Paszat)). The other components were 1) modelling benefits, risks, and cost effectiveness (12), 2) a feasibility study of an electronic pre-screening form in primary care $(13), 3$ ) a qualitative study of the views of both individuals who were long-time smokers and PCPs, and 4) the creation of a risk assessment tool.

\section{Sampling and Recruitment:}

Sampling: We aimed for maximum variation (14) by purposively sampling practicing PCPs from diverse health regions (North East, South West and Central Local Health Integration Networks (LHINs)) in Ontario, Canada and from different practice models (including fee-for-service, family health teams (FHT) and community health centres (CHC). Approximately $75 \%$ of Ontarians are patients in medical 'homes' which involve some type of capitation (15-Glazier). About half of capitation practices employ allied health professionals. All PCPs in Ontario, regardless of practice setting were eligible to receive bonuses for achieving screening targets, although the effectiveness of this incentive has been questioned (16Kiran). We also recruited Cancer Care Ontario Regional Primary Care Leads (RPCL) for a focus group. RPCLs are family physicians with a formal, recognized regional leadership role in cancer care in the province (https://archive.cancercare.on.ca/pcs/primcare/primary care network/). They are 
knowledgeable about primary care in their respective LHINs and provide continuing education to local PCPs.

Recruitment in North Eastern Ontario was by email from the RPCL for the LHIN. The recruitment of the CHC in the South West LHIN was via email by one of the research team (LP) to the site lead physician; recruitment for the FHT in the Central LHIN was by another team member (FS) to the FHT lead physician. Recruitment of the RPCLs was by email from staff at Cancer Care Ontario, the cancer organization in the province. For the FHTs and CHCs, PCPs and staff including nurse practitioners, nurses, prevention and administrative staff were invited to participate by the site lead physician.

Data Collection: An interview guide was developed by two team members (DL, MAO) and reviewed with the team. Focus groups were used to explore attitudes toward LDCT screening. Within each focus group, a modified nominal group process (17) was used to identify and prioritize information needs of PCPs. Before each focus group, participants were sent slides containing information on the current study and NLST findings(Table 1). Focus groups were held between June and October 2014, were led by an experienced qualitative researcher (DL) and co-facilitated by other team members (FS and/or MAO).

Coding and Analysis: Two team members (DL, MAO) developed a coding scheme then applied it independently to each interview transcript. NVivo software (NVivo 9, QSR International) was used for data management. Meetings were held to resolve any disagreements. Overarching themes were derived from the coded data. The data were explored for confirming and disconfirming concepts using principles of the constant comparative method (18). We reached informational saturation (19). All analytic steps were documented in an audit trail (20). In the design, we followed principles proposed by Malterud to ensure study rigor (21) including a systematic process for the analysis and examination of data for evidence of disconfirming views. We used the consolidated criteria for reporting qualitative research (22) in the preparation of the manuscript (Supplemental Table S1). 
Individual PCPs and teams were offered $\$ 200.00$ CAD as an acknowledgement of the time required for study participation. The study was approved by the North York Hospital Research Ethics Board (REB) and the University of Toronto REB.

\section{Results}

Four in-person focus groups were held with 33 PCPs and staff: 27 (82\%) female; 21 (64\%) physicians, 6 (18\%) allied health professionals (AHPs), 6 (18\%) administrative staff. AHPs included nurse practitioners, nurses, and prevention counsellors (Table 2).

Most PCPs had positive attitudes toward a potential LDCT screening service in Ontario. Physicians recounted difficult experiences with patients with Stage 4 lung cancer and hoped that screening would lead to increased early detection and treatment.

As one family physician said,

“...this is something that I'm excited about being able to do. Anyone who has been in practice for a length of time has seen these patients..., you see the spot on the x-ray or they have a symptom and you know what's coming and it's devastating to you and your patient." PCP, CHC

The detailed analysis resulted in five major themes; these are summarized below.

\section{Themes}

\section{Variable preferences for involvement of PCPs in identifying eligible patients for LDCT screening}

Several PCPs were supportive of some involvement in LDCT screening such as running searches on electronic medical records (EMRs) to identify eligible patients and by discussing pros and cons of LDCT screening with patients. These activities were similar to those undertaken by PCPs in other areas of cancer screening. As one PCP said, 
“... I think we could very well be involved because our EMRs, we target smokers and we can run searches on our EMRs to find patients, exactly the ones you're looking for, so... we do it for all the big four, colon cancer and so on." PCP Northern Ontario

While only a few PCPs thought they could implement full eligibility screening, others said they could conduct a pre-assessment for patients. For example,

"I can't speak for other doctors but l'd probably be doing it [pre-assessment] in the office myself if it was an easy sort of handout .... That way you could refer the person with the score and then it was either denied or approved based on that." PCP FHT

However, other PCPs preferred a minimal role in screening such as advertising in waiting rooms. PCPs who preferred a minimal role were concerned about increased workload associated with screening. For example,

"[In] 10 to 15 minutes I cannot see myself conscientiously committing to doing anything else ... I like our [EMR] reminders... of the PAPs, the mammograms, the faecal occult blood. This could certainly be something else that's there. But I cannot conscientiously say that I'm going to be able to do this." PCP Northern Ontario

PCPs also noted that unlike other screening programs that are largely age-based, LDCT screening for lung cancer is based on other factors such as amount smoked and duration of smoking. PCPs expressed that there was potential for confusion about eligibility for screening based on age and smoking history alone especially since smoking history may not be well-documented in the EMR.

\section{Other primary care practice professionals may have a role in LDCT screening}


Allied Health Professionals (AHPS)

PCPs indicated that AHPs -such as Nurse Practitioners, Registered Nurses, and Registered Practical Nurses- could, in certain situations play an active role in identifying eligible patients and by providing information about LDCT screening. Physicians said that AHPs could conduct brief lung cancer risk assessments for new patients. Nurses in the FHT and $\mathrm{CHC}$ groups concurred and emphasized the importance of their training and experience that would enable them to conduct risk assessments. It was noted that AHP involvement in the identification of eligible patients might not be feasible in practices with few and/or busy AHPs.

For example, a PCP who supported the involvement of AHPs in LDCT screening said,

“...for us it would be an RN [Registered Nurse] or a RNA [Registered Nursing Assistant] who's processing the patient, sees that the person's a smoker, has been for a long time, and that she would have more time.." PCP Northern Ontario

AHPs raised another positive aspect of preliminary screening by nurses such as helping patients with low literacy. For example,

"We have a lot of clientele that have barriers to learning so I don't want to get them agitated if we're asking them to do a survey and they can't read or even write... so maybe it's a good thing even if the nurses did a little bit of the survey with them..." AHP, CHC

Role of Front Desk Staff

PCPs did not think that it would not be appropriate for front desk staff to conduct the eligibility assessment of patients for LDCT screening and that staff would have a very limited role in supporting patients. Front desk staff indicated that staff training would be essential for them to understand all steps in a screening program so they could in turn, describe them to patients. Front desk staff 
emphasized their role in communicating with patients and the importance of taking a customer service approach so that patients would not be confused or misinformed about LDCT screening.

For example, a staff member said,

"Even when we have to send people down to get an x-ray or to get a MRI they hear the names but they really don't know what they're going for. They don't really understand it. " Staff member, FHT

Staff described that they were an important source of information and related several situations with other screening programs whereby patients expressed uncertainty about next steps and turned to staff for help.

\section{PCPs and AHPs have high information needs about LDCT lung cancer screening}

Across all four focus groups, PCPs and AHPs discussed their information needs about LDCT screening.

PCPs rated the following topics as high priority for information: evidence for LDCT screening including potential benefits and harms such as false positive results; program eligibility criteria including descriptions of those considered to be at high risk to develop lung cancer; suggestions for communicating risk information to patients; details of a proposed screening process; and the follow-up process for abnormal results. AHP and office staff wanted to know more about eligibility criteria and the screening process so they could provide accurate information to patients. Participants recommended that LDCT screening programs provide standardized information packages to PCPs for distribution to patients.

4. PCPs recommend that a future LDCT screening service be modelled on the current Ontario Breast Screening Program (OBSP) 
PCPs expressed that they have learned what works for their patients and their practices from experiences with three existing cancer screening programs (breast, cervical, and colorectal) and prefer the model of the OBSP. PCPs highlighted two reasons for their views: a) program communicates well; and b) program arranges follow-up for patients with positive results.

a) Program communicates well: PCPs commented positively that a strength of the OBSP was that they were kept "in the loop" of the screening process. For example,

"I, as a family doctor, would like to know that there was a $4 \mathrm{~mm}$ lesion found, it's been reviewed by a thoracic surgeon...and the recommendations are there." PCP, Northern Ontario

b) Program arranges follow-up for patients with positive results: PCPs relayed frustration with screening programs other than the OBSP when the PCP's office is responsible for contacting a specialist. PCPs described that staff often made multiple phone calls to secure a specialist appointment which often resulted in delays for patients. This frustration with follow-up was endorsed by PCPs in all groups.

For example,

"Is this gonna come back to me and say well there's an abnormal CT chest with a two centimeter nodule, have a nice day, or is it recommend $\mathrm{CT}$, three months to gauge interval, recommend biopsy immediately for suspicious lesion ? I mean how much is gonna be communicated to me to what happens when the CT doesn't come back normal?" PCP, FHT

\section{Links to smoking cessation is crucial for PCPs to support a future program}

PCPs strongly expressed that smoking cessation services should be a key component of a lung cancer screening program. PCPs were highly supportive of smoking cessation services while acknowledging that there were regional differences in service provision. PCPs perceived that a new LDCT screening program for lung cancer would provide an impetus for them to re-engage with patients who continued to smoke. 
“... this is a phenomenal opportunity for those people who are identified as not being eligible for the low dose $\mathrm{CT}$ to have a discussion regarding smoking cessation. That's absolutely crazy not to use this as an opportunity." PCP, RPCL

RPCLs explained that the effective integration of smoking cessation services into the screening process would be a key feature that will increase the acceptability of LDCT lung cancer screening among Ontario family physicians.

\section{Discussion}

Our study examined PCPs' views of their potential roles in an organized LDCT program, identified significant knowledge needs and highlighted aspects of a program that are desired based on experiences with other screening programs. We found that most PCPs were generally supportive of an organized LDCT lung cancer screening program; however, there was considerable variability in PCPs' preferences for their role. An implication of this finding is that a future organized LDCT screening program in Ontario that relies on PCPs to identify potentially eligible patients, refer them for screening, and follow up on results may not be feasible. PCPs also expressed concerns about the identification of high-risk patients in the EMR especially those who had quit smoking within the last 15 years. Greiver et al. have reported that identifying smoking history from the EMR may be difficult (23).

The current study contributes information about implementation of organized LDCT screening programs including the roles of PCPs and AHPs. As LDCT screening is being implemented outside of a trial setting, it is important to investigate the perspectives of key stakeholders including PCPs who will have some role in such a program. PCPs routinely screen patients for other types of cancers- breast, cervical, and colorectal. They have trusting relationships with patients and are well-placed to counsel them about the risks and benefits of screening. LDCT screening is known to have high false positive rates (6) and it is important that patients understand such risks and potential benefits before agreeing to participate in a 
LDCT screening program. There is emerging evidence that patient decision aids may be helpful to provide information about risks and benefits of $\operatorname{LDCT}$ screening $(24,25)$ although, participants in our study were unaware of such tools.

Several of our findings are similar to other research in primary care $(9,26-28)$. For example, Hoffman et al. described the perspectives of PCPs from New Mexico, US. In their study, several PCPs were unaware of the NLST and its application to their setting. PCPs in that study also expressed concerns about the high false positive screening rate (9). Kanodra and colleagues conducted a qualitative study of the views of patients $(n=28)$ and PCPs $(n=13)$ at a Veterans Administration medical centre. While $58 \%$ of these providers were aware of US Preventive Services Task Force screening guidelines, few providers could describe eligibility criteria. Providers indicated that shortage of time was a significant factor in limiting their ability to counsel patients about LDCT screening. They preferred that a screening coordinator review any incidental findings with patients and need for ongoing screening (26).

Our study found that PCPs preferred a specific model for a future organized LDCT screening program, in which the program arranged screening and follow up while communicating with PCPs, based on experience with an existing organized screening program for breast cancer patients that worked well for PCPs, their staff and patients.

Strengths and Limitations: We conducted our study in several health regions in Ontario, Canada and sampled PCPs from different practice types including FHTs and community health centres, and included AHP and staff in our focus groups. This is important as our results show that AHPs may have a key supportive role in a screening program and referrals to smoking cessation programs.. The extent that our findings would be similar to those in other health regions or in different practice types is unknown. Although we attempted to recruit physicians from a purely fee for service payment model, none of the volunteer participants were from this type of model and we do not know if our findings would apply to 
them.. We asked PCPs about their roles in a potential rather than actual LDCT program; however, PCPs had considerable experience with other types of organized screening programs.

\section{Conclusions}

A future Ontario LDCT lung cancer screening program should consider the variability in PCPs' preferred roles.

PCPs preferred that a future lung cancer screening program be modelled on the OBSP including follow up of positive findings, and have explicit links to smoking cessation programs.

A future program should provide information materials and learning opportunities tailored to PCPs' identified needs.

Word Count: 3198 limit: 3000 words

Acknowledgements:

We thank the primary care providers and staff who participated in the study. The study was funded by the Ontario Cancer Screening Research Network.

A brief report of the study was submitted to Cancer Care Ontario in March 2015. 


\section{References}

1. American Cancer Society www.cancer.org/cancer/non-small-cell-lung-cancer/detectiondiagnosis-staging/survival-rates.html\#references. Accessed April 13, 2018

2. Aberle DR, Adams AM, Berg CD, Black WC, Clapp JD, Fagerstrom RM, Gareen IF, Gatsonis C, Marcus PM, Sicks JD. For the National Lung Screening Trial Research Team. Reduced lung-cancer mortality with low-dose computed tomographic screening. N Engl J Med. 2011;365(5):395-409. https://doi.org/10.1056/NEJMoa1102873.

3. Field JK, Duffy SW, Baldwin DR, Brain KE, Devaraj A, Eisen T, Green BA, Holemans JA, Kavanagh T, Kerr KM, Ledson M, Lifford KJ, McRonald FE, Nair A, Page RD, Parmar MK, Rintoul RC, Screaton N, Wald NJ, Weller D, Whynes DK, Williamson PR, Yadegarfar G, Hansell DM. The UK Lung Cancer Screening Trial: a pilot randomised controlled trial of low-dose computed tomography screening for the early detection of lung cancer. Health Technol Assess. 2016 May;20(40):1-146. doi: 10.3310/hta20400.

4. Pinsky PF, Bellinger CR, Miller DP Jr. False-positive screens and lung cancer risk in the National Lung Screening Trial: Implications for shared decision-making. J Med Screen. 2017 Jan 1:969141317727771. doi:10.1177/0969141317727771.

5. Canadian Task Force on Preventive Health Care. Recommendations on screening for lung cancer CMAJ 2016. DOI:10.1503/cmaj.151421

6. Moyer VA; U.S. Preventive Services Task Force. Screening for lung cancer: U.S. Preventive Services Task Force recommendation statement. Ann Intern Med. 2014 Mar 4;160(5):330-8. doi: 10.7326/M13-2771. 
7. Wiener RS, Gould MK, Arenberg DA, Au DH, Fennig K, Lamb CR, Mazzone PJ, Midthun DE, Napoli M, Ost DE, Powell CA, Rivera MP, Slatore CG, Tanner NT, Vachani A, Wisnivesky JP, Yoon SH; ATS/ACCP Committee on Low-Dose CT Lung Cancer Screening in Clinical Practice. An official American Thoracic Society/American College of Chest Physicians policy statement: implementation of low-dose computed tomography lung cancer screening programs in clinical practice. Am J Respir Crit Care Med. 2015 Oct 1;192(7):881-91. doi: 10.1164/rccm.2015081671ST.

8. American Lung Association. Providing guidance on lung cancer screening to patients and physicians http://www.lung.org/assets/documents/lung-cancer/lung-cancer-screeningreport.pdf. Accessed April 132018.

9. Hoffman RM, Sussman AL, Getrich CM, Rhyne RL, Crowell RE, Taylor KL, Reifler EJ, Wescott PH, Murrietta AM, Saeed Al, Mishra SI. Attitudes and beliefs of primary care providers in New Mexico about lung cancer screening using low-dose computed tomography. Preventing Chronic Disease. 2015;12:E108. doi:10.5888/pcd12.150112.

10. Henderson S, DeGroff A, Richards TB, Kish-Doto J, Soloe C, Heminger C, Rohan E. A qualitative analysis of lung cancer screening practices by primary care physicians. Journal of Community Health $2011 ; 36: 949-956$.

11. Sandelowski M. Whatever happened to qualitative description? Research in Nursing \& Health , $2000,23,334-340$.

12. Ten Haaf K, Tammemägi MC, Bondy SJ, van der Aalst CM, Gu S, McGregor SE, Nicholas G, de Koning HJ, Paszat LF. Performance and cost-effectiveness of computed tomography lung cancer 
screening scenarios in a population-based setting: a microsimulation modeling analysis in Ontario, Canada. PLoS Med. 2017 Feb 7;14(2):e1002225. doi: 10.1371/journal.pmed.1002225.

13. O’Brien MA, Sullivan F, Carson A, Siddiqui R, Syed S, Paszat L. Piloting electronic screening forms in primary care to identify patients eligible for low dose CT lung cancer screening: Findings from a mixed methods study. BMC Family Practice 2017 18:95.

14. Palinkas LA, Horwitz SM, Green CA, Wisdom JP, Duan N, Hoagwood K. Purposeful sampling for qualitative data collection and analysis in mixed method implementation research. Administration and policy in mental health. 2015;42(5):533-544. doi:10.1007/s10488-013-0528y.

15. Glazier RH, Redelmeier DA. Building the patient-centered medical home in Ontario. JAMA. 2010;303(21):2186-2187.

16. Kiran T, Wilton AS, Moineddin R, Paszat L, Glazier RH. Effect of payment incentives on cancer screening in Ontario primary care. Ann Fam Med. 2014 Jul;12(4):317-23. doi: 10.1370/afm.1664.

17. McMillan SS, King M, Tully MP. How to use the nominal group and Delphi techniques. International Journal of Clinical Pharmacy. 2016;38:655-662. doi:10.1007/s11096-016-0257-x.

18. Charmaz K: Constructing grounded theory. London; Thousand Oaks, Calif.: Sage Publications, 2006.

19. Bowen G. Naturalistic inquiry and the saturation concept. Qualitative Research. 2008;8:137-152.

20. Crabtree B, Miller W. Doing Qualitative Research, 2nd Edition, London: Sage Publications, 1999

21. Malterud K. Qualitative research: standards, challenges, and guidelines. Lancet 2001; 358: 48388. 
22. Tong A, Sainsbury P, Craig J. Consolidated criteria for reporting qualitative research (COREQ): a 32-item checklist for interviews and focus groups International Journal for Quality in Health Care 2007;19:349-357

23. Greiver M, Aliarzadeh B, Meaney C, Moineddin R, Southgate CA, Barber DT, White DG, Martin KB, Ikhtiar T, Williamson T. Are we asking patients if they smoke? Missing information on tobacco use in Canadian electronic medical records. Am J Prev Med. 2015 Aug;49(2):264-8. doi: 10.1016/j.amepre.2015.01.005.

24. Volk RJ, Linder SK, Leal VB, Rabius V, Cinciripini PM, Kamath GR, Munden RF, Bevers TB. Feasibility of a patient decision aid about lung cancer screening with low-dose computed tomography. Prev Med. 2014 May;62:60-3. doi:10.1016/j.ypmed.2014.02.006. Epub 2014 Feb 8.

25. Reuland DS, Cubillos L, Brenner AT, Harris RP, Minish B, Pignone MP. A pre-post study testing a lung cancer screening decision aid in primary care. BMC Med Inform Decis Mak. 2018 Jan 12;18(1):5. doi: 10.1186/s12911-018-0582-1

26. Kanodra NM, Pope C, Halbert CH, Silvestri GA, Rice LJ, Tanner NT. Primary care provider and patient perspectives on lung cancer screening. a qualitative study. Ann Am Thorac Soc. 2016 Nov;13(11):1977-1982. PubMed PMID: 27676369.

27. Raz DJ, Wu GX, Consunji M, Nelson RA, Kim H, Sun CL, Sun V, Kim JY. The effect of primary care physician knowledge of lung cancer screening guidelines on perceptions and utilization of lowdose computed tomography. Clin Lung Cancer. 2017 Jun 1. pii: S1525-7304(17)30148-1. doi: 10.1016/j.cllc.2017.05.013.

28. Simmons VN, Gray JE, Schabath MB, Wilson LE, Quinn GP. High-risk community and primary care providers knowledge about and barriers to low-dose computed topography lung cancer 
screening. Lung Cancer. 2017 Apr;106:42-49. doi:10.1016/j.lungcan.2017.01.012. Epub 2017 Jan 31. 
Table 1. Summary of topics in low dose CT lung cancer screening slide deck

\begin{tabular}{|l|c|}
\hline Topic & Number of slides \\
\hline Purpose of focus group & 3 \\
\hline Brief description of lung cancer & 2 \\
\hline Overview of National Lung Cancer Screening Trial & 3 \\
\hline $\begin{array}{l}\text { Potential options for high risk lung cancer } \\
\text { screening program in Ontario }\end{array}$ & 3 \\
\hline Potential roles for family physicians & 1 \\
\hline $\begin{array}{l}\text { Potential costs and safety of high risk lung cancer } \\
\text { screening }\end{array}$ & 1 \\
\hline
\end{tabular}


Table 2. Number and type of participants in low dose CT lung cancer screening focus groups

\begin{tabular}{|l|c|c|c|c|}
\hline & \multicolumn{3}{|c|}{ Location $^{1}$ of Focus Group in Ontario } \\
\hline & North East & South West & Central & $\begin{array}{c}\text { Regional Primary } \\
\text { Care Leads }^{2}\end{array}$ \\
\hline $\begin{array}{l}\text { Number of practices } \\
(\mathrm{n})\end{array}$ & 3 & 1 & 1 & N/A \\
\hline Physicians (n) & 8 & 2 & 3 & 6 \\
\hline $\begin{array}{l}\text { Allied Health } \\
\text { Professionals (n) }\end{array}$ & 0 & 6 & 1 & N/A \\
\hline Staff (n) & 0 & 2 & 4 & N/A \\
\hline
\end{tabular}

1 Location refers to the Local Health Integration Network region

2 The focus group for the Regional Primary Care Leads was held in Toronto, Ontario

N/A; not applicable 